\title{
Susceptibility to Residual Inhibition Is Associated With Hearing Loss and Tinnitus Chronicity
}

Trends in Hearing

Volume 25: $1-10$

(C) The Author(s) 2021

Article reuse guidelines:

sagepub.com/journals-permissions DOI: $10.1177 / 2331216520986303$ journals.sagepub.com/home/tia

@SAGE

\author{
S. Hu ${ }^{1,2} \mathbb{D}$, L. Anschuetz', D. A. Hall ${ }^{3,4,5}$ D, M. Caversaccio' and \\ W. Wimmer ${ }^{1,2}$
}

\begin{abstract}
Residual inhibition, that is, the temporary suppression of tinnitus loudness after acoustic stimulation, is a frequently observed phenomenon that may have prognostic value for clinical applications. However, it is unclear in which subjects residual inhibition is more likely and how stable the effect of inhibition is over multiple repetitions. The primary aim of this work was to evaluate the effect of hearing loss and tinnitus chronicity on residual inhibition susceptibility. The secondary aim was to investigate the short-term repeatability of residual inhibition. Residual inhibition was assessed in 74 tinnitus subjects with 60 -second narrow-band noise stimuli in 10 consecutive trials. The subjects were assigned to groups according to their depth of suppression (substantial residual inhibition vs. comparator group). In addition, a categorization in normal hearing and hearing loss groups, related to the degree of hearing loss at the frequency corresponding to the tinnitus pitch, was made. Logistic regression was used to identify factors associated with susceptibility to residual inhibition. Repeatability of residual inhibition was assessed using mixed-effects ordinal regression including poststimulus time and repetitions as factors. Tinnitus chronicity was not associated with residual inhibition for subjects with hearing loss, while a statistically significant negative association between tinnitus chronicity and residual inhibition susceptibility was observed in normal hearing subjects (odds ratio: $0.63 ; p=.0076)$. Moreover, repeated states of suppression can be stably induced, reinforcing the use of residual inhibition for within-subject comparison studies.
\end{abstract}

\section{Keywords}

tinnitus suppression, acoustic stimulation, hidden hearing loss

Received 17 August 2020; Revised 8 December 2020; accepted I3 December 2020

Residual inhibition (RI) refers to the phenomenon of transient tinnitus loudness suppression after exposure to an acoustic stimulus (Roberts et al., 2008; Terry et al., 1983). It was first described more than 100 years ago by Spaulding (1903) and systematically studied by Feldmann in the 1970s (Feldmann, 1971). The prevalence of RI is estimated to be over $75 \%$ in subjects with tinnitus (Roberts et al., 2006; Vernon \& Meikle, 2003). In the remaining tinnitus subjects, exposure to acoustic stimuli either does not alter tinnitus perception or, in rare cases, temporarily increases tinnitus loudness (residual excitation [RE]; Sedley et al., 2012). RI can be induced by various types of stimuli, including pure-tones (Terry et al., 1983), broadband noise (Vernon \& Meikle,

\footnotetext{
'Department of Otolaryngology, Head and Neck Surgery, Bern University Hospital, Bern, Switzerland

${ }^{2}$ Hearing Research Laboratory, ARTORG Center for Biomedical Engineering Research, University of Bern, Bern, Switzerland

${ }^{3}$ National Institute for Health Research Nottingham Biomedical Research Centre, Nottingham, United Kingdom

${ }^{4}$ Hearing Sciences, Division of Clinical Neuroscience, School of Medicine, University of Nottingham, Nottingham, United Kingdom

${ }^{5}$ University of Nottingham Malaysia, Semenyih, Malaysia

Corresponding author:

W. Wimmer, Department of Otolaryngology, Head and Neck Surgery, Bern University Hospital, Inselspital, Freiburgstrasse, 3010 Bern, Switzerland.

Email: wilhelm.wimmer@insel.ch
} 
2003), narrow-band noise (Roberts et al., 2008), and amplitude modulated sounds (Reavis et al., 2012). It has been observed that both the duration and depth of RI (i.e., the degree of tinnitus loudness change) correlate with the intensity and spectrum of the acoustic stimulus. Notably, the maximum RI time increases nonlinearly as the duration of the stimulation gets longer (Terry et al., 1983). In the majority of subjects the suppression can last from a few seconds to minutes (Vernon \& Meikle, 2003) and in rare cases even up to several hours (Olsen et al., 1996; Vernon, 1981). RI has potential as a useful tool in clinic, notably as a diagnostic marker for subtyping and also as a prognostic indicator for individual responses to therapeutic acoustic stimulation. For example, the varying depth and duration of RI in individuals could enable a more refined tinnitus classification. In cases where positive RI leads to a transient tinnitus reduction, the procedure can also be used to reassure patients during the counseling process (Fournier et al., 2018).

Despite these potential clinical benefits, RI is underrepresented in the routine assessment of tinnitus patients in clinics. A reason why could be due to uncertainties in the mechanisms underlying RI, combined with the relatively long testing times. A hypothesis has been put forward that RI is a temporary reduction of hyperactive spontaneous activity or desynchronization of excessive synchronous activity at or below the level of the auditory cortex in deafferent regions caused by hearing loss (Galazyuk et al., 2017; Kahlbrock \& Weisz, 2008; Roberts et al., 2008; Sedley et al., 2012). The suppression of spontaneous activity in the inferior colliculus was reported during RI in animal experiments (Galazyuk et al., 2017). Neuroimaging studies in human subjects showed decreased delta, theta, and gamma oscillations of the auditory cortex during RI, indicating a restoration of the balance between excitatory and inhibitory neural processes (Adamchic et al., 2017; Kahlbrock \& Weisz, 2008; Roberts et al., 2015; Sedley et al., 2012). However, no change in delta and theta and a decrease in gamma oscillations was observed in the subjects during RE, suggesting a more complex mechanism behind RI and tinnitus (Sedley et al., 2012). In addition, Galazyuk et al. (2017) showed in an animal study that repeated exposures to the acoustic stimulus are associated with a gradual reduction of inferior colliculus activity. This indicates a possible habituation effect of RI that might reduce the potential benefits of RI during the counseling process implying the importance of analyzing repeatability of RI using human subjects.

As part of a study applying RI to modulate tinnitus perception (Hu et al., 2019), we wanted to identify which factors, in addition to those already known (i.e., form, frequency, intensity, and duration of the acoustic stimulus), could have a positive influence on the susceptibility of subjects to experiencing RI. Hearing loss is one of the major factors associated with tinnitus (Shargorodsky et al., 2010). Previous studies have demonstrated that the use of narrow-band stimuli targeting the hearing loss frequency (which often coincides with the tinnitus frequency spectrum) was most effective for RI (Fournier et al., 2018; Roberts et al., 2008). However, considering the presence of tinnitus in subjects with normal audiograms (Savastano, 2008), additional factors need to be considered. Fournier et al. (2018) observed shorter tinnitus chronicity in the subgroup with complete inhibition than those with partial inhibition. In addition, transcranial magnetic stimulation studies showed more effective suppression in patients with shorter chronicity (De Ridder et al., 2005; Kleinjung et al., 2007). This indicates a possible contribution of tinnitus chronicity to the modulation of tinnitus perception. Therefore, the primary aim of this study was to evaluate RI susceptibility under consideration of a hearing loss category and tinnitus chronicity. The secondary aim was to investigate whether RI can be repeatedly induced after 10 repetitions in a short-term setting.

\section{Methods}

\section{Study Design and Setting}

The presented analysis was performed using the screening data collected in an ongoing study being conducted at the Bern University Hospital, Inselspital, Bern, Switzerland (Hu et al., 2019). The study was approved by the cantonal ethics committee of Bern, Switzerland (reference number: KEK-BE 2017-02037). The participants were recruited via the outpatient clinic in our department. All participants gave written informed consent about the usage of their data before starting the screening stage. Data of the period from February 1, 2018, to February 29, 2020, were used for the analysis.

\section{Tinnitus Subjects}

The screening data of subjects meeting the following criteria were included in the analysis: (a) age $\geq 18$ years; (b) subjective tinnitus that is not fluctuating; (c) singlepitched tinnitus, perceived unilaterally, bilaterally (in both ears), or centrally (in the head); (d) no "catastrophic" tinnitus, that is, a Tinnitus Handicap Inventory (THI) score less than 76 (Newman et al., 1996), (e) no change of tinnitus form (pure-tone or noise-like) or pitch after RI stimulus presentation, and (f) no enhancement of tinnitus loudness (RE) after exposure to an acoustic stimulus. Data from subjects with bilateral tinnitus experiencing different levels of tinnitus suppression in each ear were excluded from the analysis. 


\section{Audiometry and Tinnitus Assessment}

For a detailed description of the assessment procedure and measurement setup please refer to the protocol of the accompanying study (Hu et al., 2019). As part of the screening procedure, all participants completed a questionnaire containing information on the patients' medical history, age (in years) and tinnitus chronicity (in years), the THI questionnaire, and the Hospital Anxiety and Depression Scale (HADS) questionnaire (Zigmond \& Snaith, 1983).

All psychoacoustic measurements were performed inside an acoustic chamber. To generate the acoustic stimuli, we used a custom-written Matlab script (The MathWorks Inc, v.2017b) with the PsychophysicsToolbox extension (Brainard, 1997). The stimuli were presented through an external sound card (Scarlett2i2, FocusRite) and high-definition in-ear headphones (E1001, Triple-Driver, 1MORE Inc). Calibration of the stimuli was performed using a head and torso simulator, including two ear simulators (Type 4128, Brüel \& Kjaer) and an audio analyzer (UPV Audio analyzer DC$250 \mathrm{kHz}$, Rohde \& Schwarz). For the measurement of air conduction hearing thresholds (in $\mathrm{dB}$ sound pressure level, SPL), an extended pure-tone audiometry was performed at $0.125,0.25,0.5,1,2,3,4,6,8,9,10,11,12$, and $13 \mathrm{kHz}$. The subjects also reported their tinnitus laterality (i.e., unilateral left, unilateral right, "bilateral" in both ears or "central" in the head) and form (i.e., tonal or noise-like). The tinnitus pitch (in $\mathrm{kHz}$ ) was estimated using the two-alternative forced choice methods (2AFC) in the range of 0.125 to $13 \mathrm{kHz}$, using either pure tone or third-octave band noise stimuli, depending on the tinnitus form indicated. Tinnitus loudness (in $\mathrm{dB}$ SPL) was determined by matching with an ipsilateral stimulus at the estimated tinnitus pitch. For bilateral and central tinnitus, loudness was estimated separately for each ear. The estimation was performed starting at $5 \mathrm{~dB}$ below the hearing threshold and increasing in $1 \mathrm{~dB}$ steps.

\section{Residual Inhibition Assessment}

For RI assessment, we used a 60-second third-octave band noise stimulus, whose center frequency was set to the tinnitus pitch. For improved comparability, we additionally measured the air conduction threshold (in $\mathrm{dB}$ SPL), minimum masking level (MML; in $\mathrm{dB}$ SPL) and loudness discomfort level (LDL; in dB SPL) using the RI stimulus. To avoid potential RI during MML estimation, an intermittent stimulus with a stimulation duration of 2 to 3 seconds and silent intervals of 2 to 3 seconds was used to measure MML. In case of unilateral tinnitus, the RI stimulus was presented ipsilaterally at a level $20 \mathrm{~dB}$ above the MML. Contralaterally, the stimulation level was adjusted so that it was at the same sensation level (SL) as the ipsilateral stimulus. This was achieved by adding the difference between the RI stimulus level of the tinnitus ear and the ipsilateral third-octave narrow band noise threshold to the third-octave narrow band noise threshold of the contralateral ear. In case of a bilateral or central tinnitus, both ears were stimulated with the same stimulus $20 \mathrm{~dB}$ above the MML. To assess the short-term repeatability of RI, subjects who reported suppression of their tinnitus after acoustic stimulation were repeatedly examined in 10 consecutive trials. Between the individual repetitions, the subjects used a response box to rate the change in tinnitus loudness on an 11-point Likert scale (range: -5 to $5 ;-5$ complete suppression, 0 no change, +5 enhancement) until it returned to its previous level. To assess the time-related change in RI depth, the time of each rating was recorded (denoted "RI time"). After the tinnitus loudness had returned to its baseline level, the next repetition was initiated. Our primary outcome measure of RI likelihood was the maximum RI depth after stimulus offset averaged over the 10 repetitions. Subjects who achieved an averaged maximum RI depth of -5 or -4 (corresponding to a complete or almost complete suppression of tinnitus) were assigned to the "RI group" (i.e., having RI capability), while the remaining subjects were assigned to the "Comparator" group (no substantial suppression). The conservative threshold of -4 was chosen based on the assumption that substantial RI should be observed in the subjects when using a RI stimulation level of $20 \mathrm{~dB}$ above MML. The time after which the tinnitus returned to the loudness before the stimulus (i.e., the subject presses 0 after RI) was defined as maximum RI time (in seconds). Only data of subjects from the "RI group" were included in the analysis of the short-term repeatability of RI. For analysis, the RI depth and RI time of all repetition trials were used as secondary outcome measures.

\section{Statistical Analysis}

Descriptive statistics were used to summarize demographic data, tinnitus characteristics and RI outcomes. The hearing thresholds were converted from $\mathrm{dB}$ SPL to $\mathrm{dB}$ hearing level (HL) using the reference values specified in the literature for pure-tone (reference age group: 10-21 years; Lee et al., 2012). For the hearing loss categorization, the averaged hearing threshold at the frequency corresponding to the tinnitus pitch and the two adjacent frequencies of the tinnitus ear was used (hearing loss: $>25 \mathrm{~dB}$ HL; normal hearing $\leq 25 \mathrm{~dB}$ HL). On average, the normal hearing group subjects were 25.0 years younger than the subjects in the hearing loss group (CI [20.0, 29.5]; $p<.001)$. In addition, we calculated the Pearson correlation coefficients between the 
HADS and THI questionnaires, which are known to be correlated (Andersson et al., 2009). This was confirmed by the correlation coefficients of 0.58 between THI and HADS-A responses (CI [0.37, 0.73]; $p<.001$ ), 0.57 between THI and HADS-D responses (CI [0.39, 0.70]; $p<.001$ ), and 0.66 between HADS-A and HADS-D responses (CI [0.48, 0.80]; $p<.001$ ).

To test the differences between the "RI" and "Comparator" groups for demographic and tinnitus characteristics, we applied the Mann-Whitney $\underline{\mathrm{U}}$ and $\chi^{2}$ tests for continuous and categorical variables, respectively. We used multivariable logistic regression to compute the odds ratios (ORs) for the susceptibility to substantial RI (i.e., almost complete or complete RI), with the dependent outcome variable defined as the RI group (Comparator vs. RI). The initial model was populated with effects for hearing loss category, age, gender, tinnitus form, tinnitus laterality, tinnitus chronicity, THI score, tinnitus pitch, MML, stimulation level (in dB SL). and LDL. The HADS scores were not included because of the strong collinearity with THI scores. An interaction between hearing category and tinnitus chronicity was included to model dependencies between the variables. A step-wise backward elimination based on Akaike's Information Criterion was applied for model selection, resulting a final model that consisted of hearing category, tinnitus chronicity. and the interaction term between both variables.

The short-term repeatability of RI was assessed using an ordinal mixed-effects model with RI depth (i.e., levels -5 to 0 ) as the ordinal dependent outcome. The variables RI time (time after stimulus offset) and repetition (trials 1-10) were included as fixed effects with an interaction. All other covariates showed a lack of statistical significance. The subject identity number was included as random intercept to account for repeated-measures. All statistics were performed using the $\mathrm{R}$ environment (version 3.6.2; R Core Team, 2017), with the modules to "mixor" (Archer et al., 2018) for ordinal mixed-effects model fitting.

\section{Results}

\section{Data Characteristics}

From the data set of 109 screened tinnitus subjects, the records of 74 subjects were included in the analysis (Figure 1). A summary of the data is given in Table 1. The majority of the subjects indicated a pure-tone tinnitus $(78 \%)$. Interestingly, almost three fourths of the subjects experienced their tinnitus pitch at a frequency above $8 \mathrm{kHz}$ (average tinnitus pitch of $9.2 \mathrm{kHz}$ ), that is, within a test range usually not covered in routine clinical audiometry. The mean hearing threshold at this tinnitus pitch was $40.5 \mathrm{~dB} \mathrm{HL}$, and the mean reported tinnitus loudness was 7.2 dB SL. The majority of the subjects had a tinnitus chronicity that had lasted longer than 1 year (62 of 74 in our cohort). Generally across the group, the THI and HADS scores indicated slight tinnitus symptom severity and low levels of anxiety and depression, respectively.

The proportion of subjects assigned to the "RI" and "Comparator" groups was 65\% (48/74) and 35\% (26/ 74), respectively. Just over $50 \%$ of subjects (39/74) were characterized by a hearing loss (with a maximum hearing threshold of $91.8 \mathrm{~dB}$ HL) and with a substantial RI of their tinnitus, which we observed meant that the post hoc subgroups for hearing status and RI status were

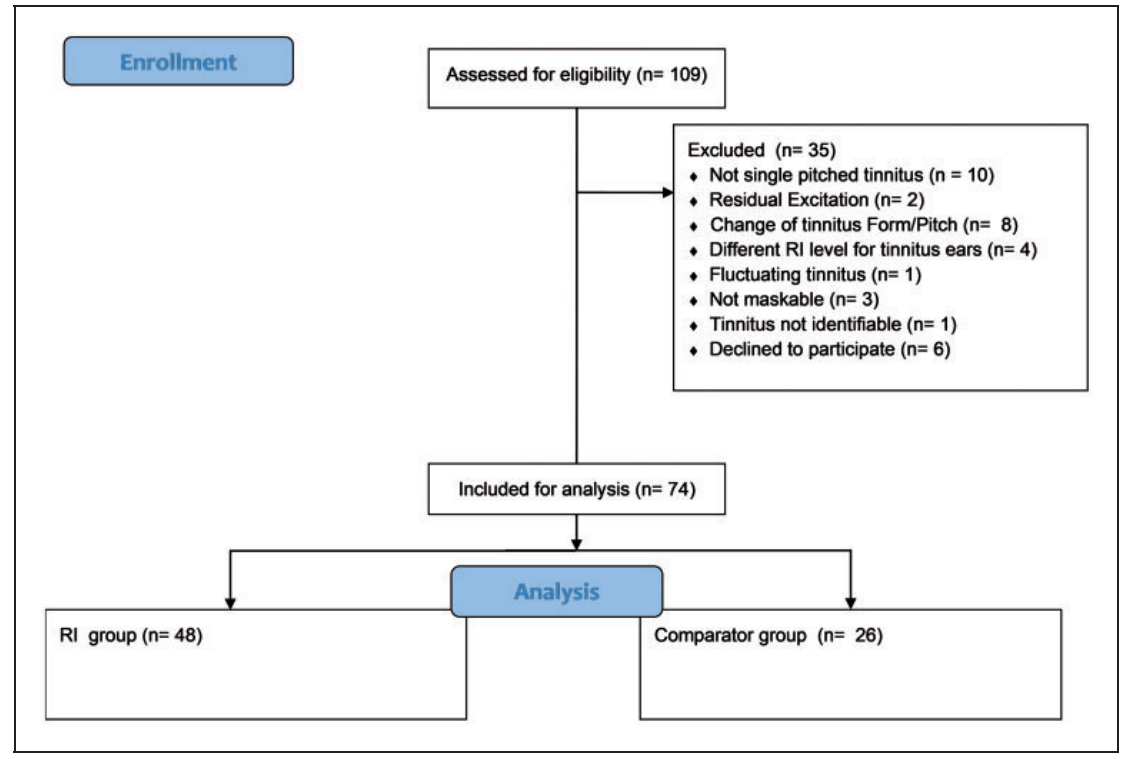

Figure I. Flowchart for Screening Data Inclusion. $\mathrm{RI}=$ residual inhibition. 
Table I. Overview of Demographic Details, Tinnitus Characteristics, and Residual Inhibition Outcomes.

\begin{tabular}{|c|c|c|c|}
\hline & Comparator $(n=26)$ & $\mathrm{RI}(n=48)$ & All $(n=74)$ \\
\hline \multicolumn{4}{|l|}{ Hearing category } \\
\hline Hearing loss group & $12(46 \%)$ & $39(81 \%)$ & $51(69 \%)$ \\
\hline Normal hearing group & $14(54 \%)$ & $9(19 \%)$ & $23(31 \%)$ \\
\hline \multicolumn{4}{|l|}{ Gender } \\
\hline Female & $9(35 \%)$ & $16(33 \%)$ & $25(34 \%)$ \\
\hline Male & $17(65 \%)$ & $32(67 \%)$ & 49 (66\%) \\
\hline Age, years & $41.8( \pm 16.1)$ & $49.7( \pm 14.1)$ & $46.9( \pm 15.2)$ \\
\hline Hearing threshold at tinnitus pitch, $\mathrm{dB} H \mathrm{HL}$ & $33.5( \pm 29.9)$ & $44.4( \pm 27.3)$ & $40.5( \pm 28.5)$ \\
\hline Hearing threshold (PTA), dB HL & $14.3( \pm 13.6)$ & $17.6( \pm 15.2)$ & $16.4( \pm \mid 4.6)$ \\
\hline Tinnitus chronicity, years & $8.9( \pm 7.0)$ & $10.0( \pm 10.2)$ & $9.6( \pm 9.2)$ \\
\hline \multicolumn{4}{|l|}{ Tinnitus form } \\
\hline Noise-like & $7(27 \%)$ & $9(19 \%)$ & $16(22 \%)$ \\
\hline Pure-tone & $19(73 \%)$ & $39(81 \%)$ & $58(78 \%)$ \\
\hline \multicolumn{4}{|l|}{ Tinnitus laterality } \\
\hline Bilateral & $10(38 \%)$ & $21(44 \%)$ & 31 (42\%) \\
\hline Central & $6(23 \%)$ & $9(19 \%)$ & $15(20 \%)$ \\
\hline Unilateral & $10(38 \%)$ & $18(38 \%)$ & $28(38 \%)$ \\
\hline Tinnitus pitch, kHz & $10.0( \pm 2.1)$ & $8.7( \pm 3.1)$ & $9.2( \pm 2.8)$ \\
\hline Tinnitus loudness, dB SL & $7.2( \pm 7.9)$ & $7.3( \pm 9.0)$ & $7.2( \pm 8.6)$ \\
\hline Minimum masking level, dB SL & $16.5( \pm 12.0)$ & $16.6( \pm 12.3)$ & $16.6( \pm 12.1)$ \\
\hline Loudness discomfort level, dB SL & $47.1( \pm 14.7)$ & $45.4( \pm 15.7)$ & $46.0( \pm 15.3)$ \\
\hline THI score & $28.7( \pm 20.3)$ & $28.8( \pm 20.3)$ & $28.8( \pm 20.2)$ \\
\hline HADS-A score & $4.7( \pm 3.8)$ & $5.2( \pm 3.1)$ & $5.0( \pm 3.3)$ \\
\hline HADS-D score & $3.1( \pm 3.3)$ & $3.8( \pm 3.3)$ & $3.5( \pm 3.3)$ \\
\hline Averaged maximum RI depth & $-1.3( \pm 1.6)$ & $-4.8( \pm 0.3)$ & $-3.5( \pm 2.0)$ \\
\hline Averaged maximum RI time, seconds & $21.8( \pm 29.1)$ & $93.3( \pm 49.4)$ & $67.5( \pm 55.1)$ \\
\hline
\end{tabular}

Note. Comparator = subjects with no substantial residual inhibition (RI depth $>-4$ ); RI = subjects with (almost) complete residual inhibition (RI depth $\leq-4) ; \mathrm{HL}=$ hearing level; PTA = pure-tone average over 0.5, I, 2, 4, and $8 \mathrm{kHz}$; THI = Tinnitus Handicap Inventory; HADS = Hospital Anxiety and Depression Scale; $\mathrm{SL}=$ sensation level. Continuous variables are summarized with their mean values ( \pm standard deviation).

not balanced. In the "Comparator" group, 15 of 26 subjects did not experience any RI (averaged maximum RI depth $>-1)$, while the remaining subjects experienced partial RI (averaged maximum RI depth $>-4$ and $\leq-1$ ). Two of the 48 subjects in the RI group experienced long-term RI (maximum RI time $\geq 5$ minutes). Since the maximum RI time could not be measured within the time available in the screening session, these two subjects were excluded from the descriptive statistics for the maximum RI time.

\section{Susceptibility to Residual Inhibition}

The demographic results from Table 1 showed a higher percentage of "Comparator" subjects in the group with normal hearing (14 out of 23) than in the group with hearing loss (12 out of 51). The comparison between "RI" and "Comparator" in different hearing categories calculated with $\chi^{2}$ tests revealed a statistically significant difference $(p=.0043)$ indicating that subjects with hearing loss at their tinnitus frequency are more susceptible to RI. In addition, with the exception of age, which showed a trend toward younger subjects in the "Comparator" group (age difference of -8.5 years, CI
$[-18.1,-0.02] ; p=.049)$, no statistically significant differences between the groups were observed in the other characteristics tested.

The results of the logistic regression analysis for the RI susceptibility are presented in Table 2 . Tinnitus chronicity for hearing loss group did not have a statistically significant effect on RI susceptibility. However, statistically significant ORs for tinnitus chronicity for normal hearing group were observed. For a 1-year increment in tinnitus chronicity, the probability for RI susceptibility decreased by a factor of 0.63 (CI [0.41, 0.83]; $p=.0076$ ). These results suggest that tinnitus chronicity only affects the RI susceptibility for subjects with hearing thresholds at their tinnitus frequency $\leq 25 \mathrm{~dB}$ HL. Moreover, subjects with shorter tinnitus chronicity are more susceptible to RI.

\section{Short-Term Repeatability of Residual Inhibition}

In general, the RI depth and course of recovery were stable over the 10 repetitions for each individual. Statistically significant effects were observed for RI time, repetition, and their interaction term (see Table 3). Obviously, the chance for stronger suppression 
Table 2. Logistic Regression Odds Ratios With Respect to the "Comparator" Group (i.e., No Substantial Residual Inhibition).

\begin{tabular}{lllll}
\hline & & \multicolumn{2}{c}{ Confidence interval } \\
\cline { 3 - 4 } & Odds ratio & $2.5 \%$ & $97.5 \%$ & .27 \\
\hline (Intercept) & 2.21 & 0.85 & .12 \\
Hearing category (normal hearing) & 2.86 & 0.40 & 32.27 & .33 \\
Tinnitus chronicity & 1.07 & 0.98 & 1.23 & .19 \\
Hearing category (normal hearing): tinnitus chronicity & 0.63 & 0.41 & 0.83 & .0076 \\
\hline
\end{tabular}

Table 3. Mixed-Effects Ordinal Regression Odds Ratios With Respect to the RI Depth Level 0 (Return of Tinnitus Loudness to the Prestimulus Level).

\begin{tabular}{|c|c|c|c|c|}
\hline & \multirow[b]{2}{*}{ Odds ratio } & \multicolumn{2}{|c|}{ Confidence interval } & \multirow[b]{2}{*}{$p$} \\
\hline & & $2.5 \%$ & $97.5 \%$ & \\
\hline (Intercept) & 1.57 & 0.77 & 3.22 & .21 \\
\hline \multicolumn{5}{|l|}{ RI depth } \\
\hline Level -4 & 6.50 & 5.85 & 7.22 & $<.001$ \\
\hline Level -3 & 30.63 & 27.18 & 34.52 & $<.001$ \\
\hline Level -2 & 135.83 & 117.18 & 157.45 & $<.001$ \\
\hline Level -I & 762.38 & 682.08 & 852.14 & $<.001$ \\
\hline RI time & 0.93 & 0.93 & 0.94 & $<.001$ \\
\hline Repetition & 1.08 & 1.05 & I.II & $<.001$ \\
\hline RI time: repetition & 0.9989 & 0.9986 & 0.9993 & $<.001$ \\
\hline Subject ID (random intercept) & 10.11 & 2.28 & 44.77 & .0023 \\
\hline
\end{tabular}

Note. $\mathrm{RI}=$ residual inhibition.

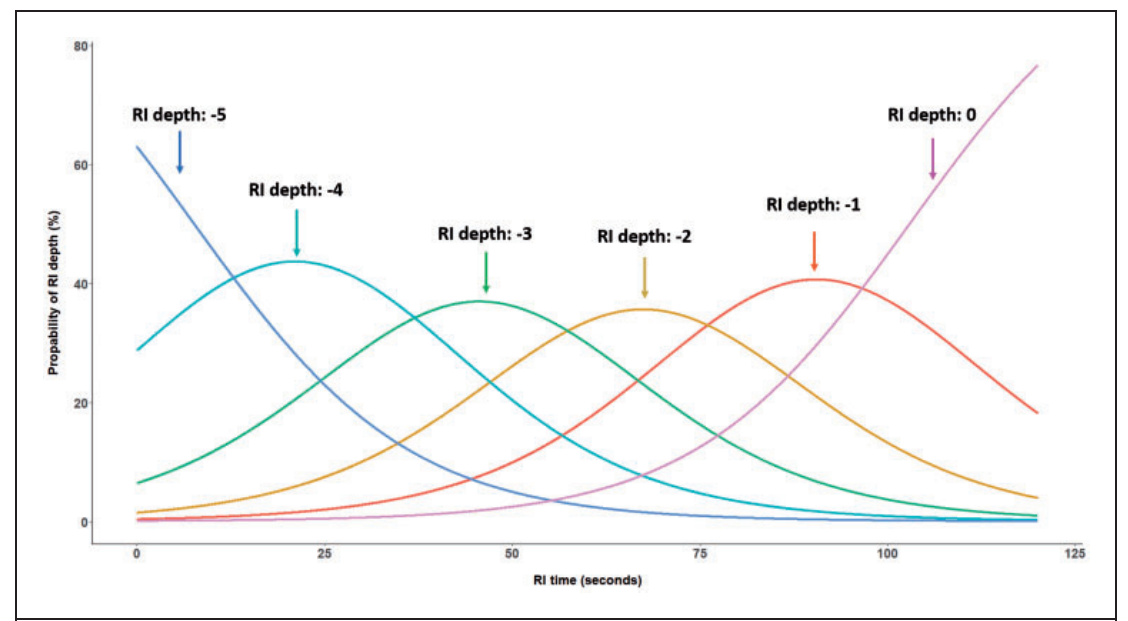

Figure 2. Probability of Experiencing a Residual Inhibition (RI) Depth Level Between -5 (Complete Suppression of Tinnitus) and 0 (Return of Tinnitus Loudness to the Initial Level) After Stimulus Offset for the First Repetition. Subjects with substantial RI ( $n=46)$ were included in the analysis.

decreases after stimulus offset (i.e., for longer RI times). Figure 2 illustrates the probability of reaching the different RI depth levels ( -5 to 0 ) for the first repetition as a function of RI time. Approximately 100 seconds after stimulation offset, the majority of subjects will either perceive their tinnitus with a slight suppression
(RI depth level -1) or its initial loudness (RI depth level 0). Moreover, the more repetitions are performed, the higher the probability to experience complete RI (i.e., an RI depth level of -5 ). Figure 3 illustrates the effect of the interaction term between RI time and repetition. After 10 repetitions, the probability of a 


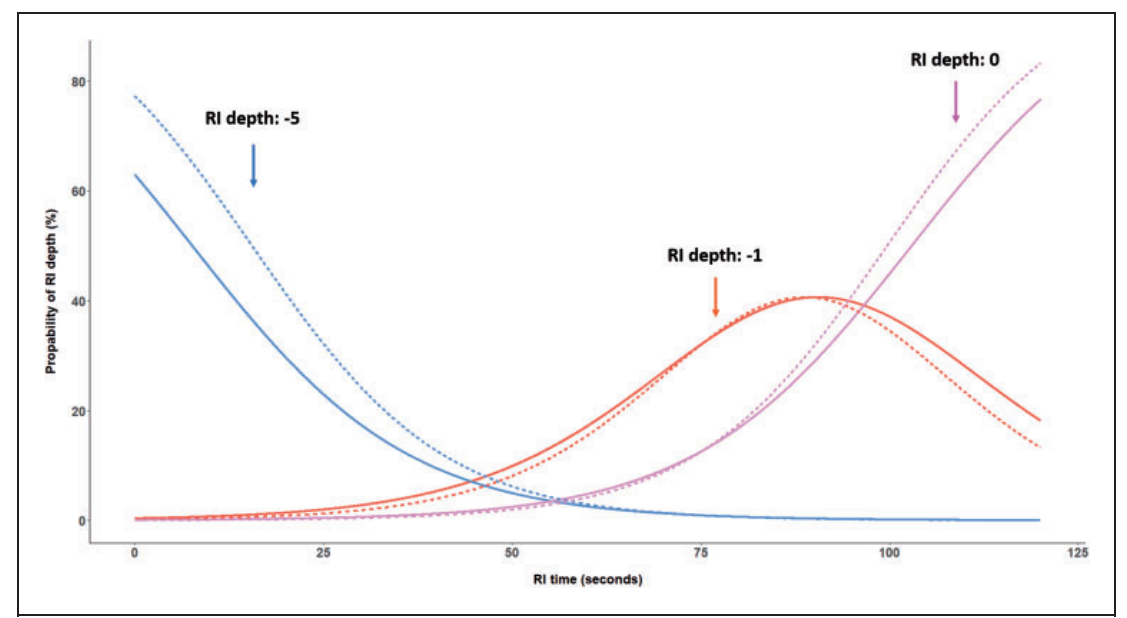

Figure 3. Probability of Experiencing Residual Inhibition (RI) depth levels of -5 (Complete Suppression), $-\mathrm{I}$ (Weak Suppression), and 0 (Return to Initial Tinnitus Loudness) After Stimulus Offset for the Ist (Solid Lines) and the IOth Repetition (Dashed Lines).

maximum RI depth of -5 increases, while the maximum RI time (i.e., return to RI depth 0 ) occurs slightly earlier. We conducted this initial analysis on a homogeneous group comprising only those 46 (i.e., 46 of 48 subjects with complete RI and a maximum RI time of less than 5 minutes) subjects with substantial RI. To explore the robustness of this pattern of findings, we took the approach of a sensitivity analysis by including the data for the 11 subjects experiencing partial RI. The pattern of results for repeatability of RI was similar to those for the subjects with substantial RI. This suggests that with the given conditions used during our assessment (i.e., 60 seconds stimulus, 10 repetitions, stimulus level at MML $+20 \mathrm{~dB}$ ) stable repeated RI phenomena can be generated.

\section{Discussion}

The main finding of our study is that tinnitus chronicity is negatively associated with RI susceptibility in subjects with normal hearing thresholds at their tinnitus frequency. In addition, the tinnitus tends to be more susceptible to transient modulation in subjects with hearing loss than in normal hearing subjects. In combination with the observed negative influence of chronicity in the normal hearing group, the difference in RI susceptibility based on hearing categorization may enable refined tinnitus subtyping. The higher susceptibility to RI in the hearing loss group suggests a higher weight of peripherally caused tinnitus leading to neuroplastic changes in the auditory system, while the influence of chronicity in the normal hearing group indicates the maintenance of tinnitus involving a more complex network of both auditory and non-auditory systems. Furthermore, we found that consecutive repetitions of acoustic stimulation provide stable RI conditions. This finding validates that RI can be used to induce repeated states with or without tinnitus in the same subject, which is important in the context of within-subject comparison studies (e.g., $\mathrm{Hu}$ et al., 2019). The prevalence of RI observed in our study, that is, 58 of 74 subjects $(78 \%)$ with some degree of residual inhibition and 48 subjects $(65 \%)$ with substantial tinnitus suppression, is comparable to previous studies reporting a prevalence between $61.5 \%$ and $88.0 \%$ (Deklerck et al., 2019; Henry \& Meikle, 2000; Roberts et al., 2008; Vernon \& Meikle, 2003). The mean maximum RI time of 93.3 seconds is comparable to the findings of Vernon and Meikle (2003). In our cohort, $78 \%$ of the participants indicated a tinnitus pitch equal or higher than $9 \mathrm{kHz}$, emphasizing the importance of extended audiometry in the clinical routine assessment of tinnitus.

\section{Susceptibility to Residual Inhibition}

The current assumption of the underlying mechanism is that RI is produced by neuronal changes in excessive activity at peripheral or central levels caused by hearing loss following acoustic stimulation in the deafferent regions (Fournier et al., 2018; Roberts et al., 2008). Previous studies have shown that particular characteristics of the acoustic stimulus targeting tinnitus and hearing lesions are known to influence the depth and duration of RI. Terry et al. (1983) observed that the maximum RI time increases in a logarithmic fashion with increasing stimulus duration. Moreover, it is known that an acoustic stimulation resembling the hearing loss that often coincides with the tinnitus spectrum induces RI more effectively (Fournier et al., 2018; Roberts et al., 2008). However, despite the fact that hearing loss is one of the main factors contributing to tinnitus, it is not a necessary condition. In addition to hearing loss, tinnitus chronicity has been shown to be a 
contributory factor in the pathology of tinnitus. Vanneste et al. (2011) demonstrated a change in activity in several brain areas and in functional connectivity between auditory and nonauditory brain structures over time. Other studies observed a correlation of tinnitus chronicity with frontostriatal connectivity (nucleus accumbens-ventromedial prefrontal cortex; Hullfish et al., 2019) and thalamic functional connectivity to different brain regions in normal hearing subjects (Zhang et al., 2015). In addition, in previous studies, changes in neuronal activity in both auditory and nonauditory brain areas were observed during RI.

A limitation of this post hoc categorization of "RI" and "Comparator" groups is the unequal number of subjects with and without hearing loss. We observed a trend that younger subjects are less susceptible to RI and subjects with normal hearing thresholds are generally younger in our cohort. In this study, we did not distinguish between noise-induced and age-related hearing loss, which may interact differently with the RI mechanism. Further investigations with stratified sampling need to be conducted to achieve balanced numbers across the "RI" and "Comparator" groups. In addition, this study estimated only LDL at the tinnitus frequency using narrowband third-octave noise. Although no statistically significant difference in LDL was observed between the "RI" and "Comparator" groups, hyperacusis is a factor that might affect the RI mechanisms.

\section{Short-Term Repeatability of Residual Inhibition}

Previous studies demonstrated that RI can be consistently reproducible between sessions indicating that there is no long-term adaptation affecting test-retest assessment (Deklerck et al., 2019; Roberts et al., 2008). However, the effect of consecutive repeated stimulation on shortterm adaptation and the robustness of RI has not yet been comprehensively studied in human subjects. The results of the ordinal mixed-effects model showed an increased probability for a reduced maximum RI time after several repetitions. In an animal study, a shortening of the suppression time of spontaneous firing rates of the inferior colliculus after consecutive stimulation (Galazyuk et al., 2017) was observed. Similarly, a study with a single human subject reported the reduction of the maximum RI duration after repeated stimulation (Sedley et al., 2015). We also observed an effect of repetitions on the RI depth, with a tendency to experience stronger suppression after more repetitions. In summary, our results suggest that the subjects in our study experienced stronger RI depths, however, slightly shorter maximum RI times with an increasing number of repetitions. Nevertheless, the low magnitude of the effects suggests stable RI after repeated stimulation. Our analysis demonstrates that with the test conditions applied in our assessment procedure (i.e., 60 seconds stimulus, 10 repetitions, RI stimulus level at MML $+20 \mathrm{~dB}$ ) stable repeated RI phenomena can be induced. In addition to its use in comparative withinsubject studies, the stability of RI, with its ability to modulate tinnitus perception, indicates potential applications during the therapeutic counseling process.

\section{Speculative Interpretation}

Our findings raise an interesting speculation in the context of tinnitus management strategies. The difference in RI susceptibility between hearing impaired subjects and subjects with normal hearing thresholds implies a refined tinnitus subtyping. Subjects with hearing lesions may be more susceptible to therapeutic benefits by means of acoustic stimulation targeting the deafferent regions (i.e., use of hearing aids), while strategies aiming at normalizing maladaptive neuroplasticity and aberrant neural connectivity between different brain structures that have been developed over time (i.e., use of neuromodulation techniques) might be more suitable for subjects without hearing lesions. In addition, tinnitus subjects with normal hearing thresholds and RI susceptibility could represent a group with their neural activity being susceptible to modulation, which could indicate a higher likelihood to benefit from interventions.

Another interesting perspective for the interpretation of our observations could be provided by the Bayesian brain hypothesis. It suggests that perception is an inference between sensory input and internal predictions in the brain. Sedley et al. (2016) proposed that aberrant sensory input caused by deafferentation could lead to tinnitus and that the persistence of tinnitus is caused by changing the default prediction from "silence" to "tinnitus" after a certain time of tinnitus onset. RI could therefore be represented either as a temporary normalization of abnormal sensory input, reflected in changes in spontaneous activity along the auditory pathway, or as a temporary resetting of the default prediction of "silence," reflected in neuroplasticity and connectivity in different parts of the brain. Applied to our findings, we speculate that these two mechanisms have a synergistic effect on RI, but with different weighting in the individual subjects. Subjects with hearing loss may be more inclined to adjust the abnormal sensory input after acoustic stimulation, while subjects with normal hearing may have less abnormal sensory input and therefore be more affected by the default prediction. Assuming that the volatility of the belief in a default "tinnitus" prediction continuously decreases over time, the default prediction could become less changeable with longer chronicity. 


\section{Conclusion}

The different dependency on tinnitus chronicity of RI susceptibility between tinnitus patients with and without hearing loss implies the possibility that two different RI mechanisms that act synergistically, but with different weightings. The lower RI susceptibility in subjects with normal audiograms suggests the importance of using different therapeutic interventions targeting the auditory and non-auditory systems, depending on the tinnitus subtyping. Furthermore, by excluding hearing lesions, it is assumed that subjects with RI, indicating a stronger susceptibility in tinnitus modulation, could benefit more from an intervention. In addition, we demonstrated that RI robust mechanism for generating repeated states with and without tinnitus, as required for within-subject comparison studies.

\section{Declaration of conflicting interests}

The authors declared no potential conflicts of interest with respect to the research, authorship, and/or publication of this article.

\section{Funding}

The authors disclosed receipt of the following financial support for the research, authorship, and/or publication of this article: This study was in part funded by the Infrastructure Grant of the University of Bern, Bern, Switzerland and Bernafon AG, Bern, Switzerland.

\section{ORCID iDs}

S. Hu (D) https://orcid.org/0000-0003-1392-2357

D. A. Hall (D) https://orcid.org/0000-0002-3804-1452

\section{References}

Adamchic, I., Toth, T., Hauptmann, C., Walger, M., Langguth, B., Klingmann, I., \& Tass, P. A. (2017). Acute effects and after-effects of acoustic coordinated reset neuromodulation in patients with chronic subjective tinnitus. NeuroImage: Clinical, 15, 541-558. https://doi.org/10. 1016/j.nicl.2017.05.017

Andersson, G., Freijd, A., Baguley, D. M., \& Idrizbegovic, E. (2009). Tinnitus distress, anxiety, depression, and hearing problems among cochlear implant patients with tinnitus. Journal of the American Academy of Audiology, 20(5), 315-319. https://doi.org/10.3766/jaaa.20.5.5

Archer, K. J., Hedeker, D., Nordgren, R., \& Gibbons, R. D. (2018). mixor: Mixed-effects ordinal regression analysis. $\mathrm{R}$ package version 1.0.4. $\mathrm{R}$ Foundation for Statistical Computing.

Brainard, D. H. (1997). The psychophysics toolbox. Spatial Vision, 10(4): 433-436. https://doi.org/10.1163/ $156856897 \times 00357$

De Ridder, D., Verstraeten, E., Van der Kelen, K., De Mulder, G., Sunaert, S., Verlooy, J., Van de Heyning, P., \& Moller, A. (2005). Transcranial magnetic stimulation for tinnitus:
Influence of tinnitus duration on stimulation parameter choice and maximal tinnitus suppression. Otology \& Neurotology, 26(4), 616-619. https://doi.org/10.1097/01. mao.0000178146.91139.3c

Deklerck, A. N., Degeest, S., Dhooge, I. J., \& Keppler, H. (2019). Test-retest reproducibility of response duration in tinnitus patients with positive residual inhibition. Journal of Speech, Language, and Hearing Research, 62(9), 3531-3544. https://doi.org/10.1044/2019_jslhr-h-18-0514

Feldmann, H. (1971). Homolateral and contralateral masking of tinnitus by noise-bands and by pure tones. Audiology, 10(3), 138-144. https://doi.org/10.3109/00206097109072551

Fournier, P., Cuvillier, A. F., Gallego, S., Paolino, F., Paolino, M., Quemar, A., Londero, A., \& Norena, A. (2018). A new method for assessing masking and residual inhibition of tinnitus. Trends in Hearing, 22. https://doi.org/10.1177/ 2331216518769996

Galazyuk, A., Voytenko, S., \& Longenecker, R. (2017). Longlasting forward suppression of spontaneous firing in auditory neurons: Implication to the residual inhibition of tinnitus. Journal of the Association for Research in Otolaryngology, 18(2), 343-353. https://doi.org/10.1007/ s10162-016-0601-9

Henry, J. A., \& Meikle, M. B. (2000) Psychoacoustic measures of tinnitus. Journal of the American Academy of Audiology, 11(3), 138-155.

Hu, S., Anschuetz, L., Huth, M. E., Sznitman, R., Blaser, D., Kompis, M., Hall, D. A., Caversaccio, M., \& Wimmer, W. (2019). Association between residual inhibition and neural activity in patients with tinnitus: Protocol for a controlled within-and between-subject comparison study. JMIR Research Protocols, 8(1), e12270. https://doi.org/10.2196/ 12270

Hullfish, J., Abenes, I., Yoo, H. B., De Ridder, D., \& Vanneste, S. (2019). Frontostriatal network dysfunction as a domain-general mechanism underlying phantom perception. Human Brain Mapping, 40(7), 2241-2251. https://doi. org/10.1002/hbm.24521

Kahlbrock, N., \& Weisz, N. (2008). Transient reduction of tinnitus intensity is marked by concomitant reductions of delta band power. BMC Biology, 6(1), 4. https://doi.org/10. 1186/1741-7007-6-4

Kleinjung, T., Steffens, T., Sand, P., Murthum, T., Hajak, G., Strutz, J., Langguth, B., \& Eichhammer, P. (2007). Which tinnitus patients benefit from transcranial magnetic stimulation? Otolaryngology-Head and Neck Surgery, 137(4), 589-595. https://doi.org/10.1016/j.otohns.2006.12.007

Lee, J., Dhar, S., Abel, R., Banakis, R., Grolley, E., Lee, J., Zecker, S., \& Siegel, J. (2012). Behavioral hearing thresholds between 0.125 and $20 \mathrm{kHz}$ using depthcompensated ear simulator calibration. Ear and Hearing, 33(3), 315. https://doi.org/10.1097/aud.0b013e31823d7917

Newman, C. W., Jacobson, G. P., \& Spitzer, J. B. (1996). Development of the Tinnitus Handicap Inventory. Archives of Otolaryngology-Head \& Neck Surgery, 122(2), 143-148. https://doi.org/10.1001/archotol.1996. 01890140029007

Olsen, S., Nielsen, L., Osterhammel, P., Rasmussen, A., Ludvigsen, C., \& Westernmann, S. (1996). Experiments 
with sweeping pure tones for the inhibition of tinnitus. Journal of Audiological Medicine, 5, 27-37.

$\mathrm{R}$ Core Team. (2017). R: A language and environment for statistical computing. R Foundation for Statistical Computing. https://www.R-project.org/.

Reavis, K. M., Rothholtz, V. S., Tang, Q., Carroll, J. A., Djalilian, H., \& Zeng, F. G. (2012). Temporary suppression of tinnitus by modulated sounds. Journal of the Association for Research in Otolaryngology, 13(4), 561-571. https://doi. org/10.1007/s10162-012-0331-6

Roberts, L. E., Bosnyak, D. J., Bruce, I. C., Gander, P. E., \& Paul, B. T. (2015). Evidence for differential modulation of primary and nonprimary auditory cortex by forward masking in tinnitus. Hearing Research, 327, 9-27. https://doi.org/ 10.1016/j.heares.2015.04.011

Roberts, L. E., Moffat, G., Baumann, M., Ward, L. M., \& Bosnyak, D. J. (2008). Residual inhibition functions overlap tinnitus spectra and the region of auditory threshold shift. Journal of the Association for Research in Otolaryngology, 9(4), 417-435. https://doi.org/10.1007/s10162-008-0136-9

Roberts, L. E., Moffat, G., \& Bosnyak, D. J. (2006). Residual inhibition functions in relation to tinnitus spectra and auditory threshold shift. Acta Oto-Laryngologica, 126 (Suppl. 556), 27-33. https://doi.org/10.1080/03655230600895358

Savastano, M. (2008). Tinnitus with or without hearing loss: Are its characteristics different? European Archives of OtoRhino-Laryngology, 265(11), 1295-1300. https://doi.org/10. 1007/s00405-008-0630-z

Sedley, W., Friston, K. J., Gander, P. E., Kumar, S., \& Griffiths, T. D. (2016). An integrative tinnitus model based on sensory precision. Trends in Neurosciences, 39(12), 799-812. https://doi.org/10.1016/j.tins.2016.10.004

Sedley, W., Gander, P. E., Kumar, S., Oya, H., Kovach, C. K., Nourski, K. V., Kawasaki, H., Howard, M. A., III, \& Griffiths, T. D. (2015). Intracranial mapping of a cortical tinnitus system using residual inhibition. Current Biology, 25(9), 1208-1214. https://doi.org/10.1016/j.cub.2015.02.075
Sedley, W., Teki, S., Kumar, S., Barnes, G. R., Bamiou, D. E., \& Griffiths, T. D. (2012). Single-subject oscillatory gamma responses in tinnitus. Brain, 135(10), 3089-3100. https://doi. org/10.1093/brain/aws220

Shargorodsky, J., Curhan, G. C., \& Farwell, W. R. (2010). Prevalence and characteristics of tinnitus among us adults. The American Journal of Medicine, 123(8), 711-718. https:// doi.org/10.1016/j.amjmed.2010.02.015

Spaulding, A. (1903). Tinnitus, with a plea for its more accurate musical notation. Archives of Otolaryngology, 32, 263-272.

Terry, A., Jones, D., Davis, B., \& Slater, R. (1983). Parametric studies of tinnitus masking and residual inhibition. British Journal of Audiology, 17(4), 245-256. https://doi.org/10. $3109 / 03005368309081485$

Vanneste, S., Van de Heyning, P., \& De Ridder, D. (2011). The neural network of phantom sound changes over time: A comparison between recent-onset and chronic tinnitus patients. European Journal of Neuroscience, 34(5), 718-731. https://doi.org/10.1111/j.1460-9568.2011.07793.x

Vernon, J. A., \& Meikle, M. B. (2003). Tinnitus: Clinical measurement. Otolaryngologic Clinics of North America, 36(2), 293-305.

Vernon, J. A. (1981). Some observations on residual inhibition. In M. M. Paparella \& W. L. Meyerhoff (Eds.), Sensorineural hearing loss, vertigo and tinnitus (Ear clinics international) (Vol. 1, pp. 138-144). Williams \& Wilkins.

Zhang, J., Chen, Y. C., Feng, X., Yang, M., Liu, B., Qian, C., Wang, J., Salvi, R., \& Teng, G. J. (2015). Impairments of thalamic resting-state functional connectivity in patients with chronic tinnitus. European Journal of Radiology, 84(7), 1277-1284. https://doi.org/10.1016/j.ejrad.2015.04. 006

Zigmond, A. S., \& Snaith, R. P. (1983) The Hospital Anxiety and Depression Scale. Acta Psychiatrica Scandinavica, 67(6), 361-370. https://doi.org/10.1111/j.1600-0447.1983. tb09716.x 